\section{Prevalence and Clinical Significance of Anti-DFS70 in Antinuclear Antibody (ANA)-positive Patients Undergoing Routine ANA Testing in a New Zealand Public Hospital}

\section{To the Editor:}

The term antinuclear antibodies (ANA) originally referred to autoantibodies directed against nuclear antigens and antigens in the cell cytoplasm or membrane ${ }^{1}$. The presence of elevated ANA is considered as the hallmark diagnostic test for systemic autoimmune rheumatic diseases (SARD). In most New Zealand (NZ) laboratories, ANA are detected by indirect immunofluorescence test (IIF) on HEp-2. However, fluorescent patterns are sometimes difficult to interpret ${ }^{2,3}$. Recent advances in autoimmune technologies have emerged for ANA testing, and laboratories in NZ are moving into acquiring the required knowledge and skills. In our laboratory, ANA screening slides are interpreted by a NOVA View automated IIF slide reader (INOVA Diagnostics Inc.), which incorporates a digital analysis image system, pattern recognition algorithms, and preset cutoff values. Problems still exist for the laboratory community to determine whether this system efficiently identifies antigens of clinical significance and whether the different automated systems have an appropriate level of pattern recognition agreement ${ }^{4}$.

The extractable nuclear antigen (ENA) panel is a test performed as a second stage testing for any positive ANA screen, which includes antibodies to dsDNA, Ro (SSA), La (SSB), Jo1, Scl-70, Sm, and RNP. ENA testing possesses limitations where the sensitivity and specificity vary depending on the underlying autoimmune disease $e^{5,6}$

The presence of anti-dense fine speckled (DFS) 70 could be used as a differential marker for $\mathrm{SARD}^{7,8}$. When present without any other specific ANA, they could be used to exclude a SARD diagnosis or at least infer that it is highly unlikely. The aim of our study was to determine the prevalence of anti-DFS70 in ANA-positive patients either suspected of having SARD or having known SARD, and the possibility of implementing an algorithm incorporating anti-DFS70 as a differential marker for ANA-positive patients. Ethical approval by the New Zealand Health and Disability Ethics Committee, the Waitemata District Health Board (WDHB) Ethics Committee, and the WDHB Maori Ethics Committee was granted on July 15, 2015 (reference number 15/CEN/103).

All serum samples were tested for the presence of ANA by an IIF assay (INOVA Diagnostics) and an ELISA assay (Bio-Rad). There were 211
ANA-positive serum samples selected based on either the automated ANA HEp-2 IIF or the Enzyme Immunoassay (EIA) ANA Screening Test (Bio-Rad) technique. Determination of positive or negative was defined by a preset cutoff of light intensity units at 48 for the HEp-2 slides. The qualitative EIA ANA screening was performed on a fully automated EVOLISTM System (Bio-Rad) and a calculated optical density $\geq 1.0$ was considered positive.

All positive samples were then further tested for the presence of anti-DFS70 antibodies by QUANTA Flash DFS70 CIA (INOVA Diagnostics). A value of $\geq 20$ chemiluminescent units was considered positive. Samples with positive ANA screen were subjected to ENA panel screening. The detection of specific ENA was performed using the Autoimmune EIA ANA Profile Test (Bio-Rad). Data were statistically evaluated using SAS software (Version 9.4).

Among the 211 positive ANA samples, 102 were known patients with SARD and 109 were patients without SARD. Anti-DFS70 antibodies were detected in 8 samples, $7 / 109$ (7\%) of the non-SARD ANA-positive patients and $1 / 102(0.98 \%)$ of the SARD ANA-positive patients. The prevalence of anti-DFS70 (Table 1) was significantly higher in patients without SARD compared to patients with SARD $(\mathrm{p}=0.0401)$.

The 7 positive cases of anti-DFS70 antibodies were distributed between the speckled $(1 / 88,5 \%)$, centromere $(2 / 11,18 \%)$, and homogeneous patterns $(4 / 82,5 \%)$. Anti-DFS70 was also present in 1 sample showing a mixed homogenous/centromere IIF pattern. Anti-DFS70 was the sole ANA present in 5/8 (62.5\%) samples. Other specific ANA detected were antibodies to SSA and Scl-70, as well as anticentromere antibodies; however, none of these patients presented with a SARD condition. The patients with SARD had no other specific ANA present, and did not have a history of specific ANA.

According to our results, the presence of anti-DFS70 in ANA-positive patients makes a SARD diagnosis highly unlikely, particularly when no ENA are also present. This suggests that anti-DFS70 assays can be incorporated into the ANA test algorithm. We propose 2 algorithms that could be used for cost effectiveness and diagnosis of SARD. The first algorithm is based on the topographic distribution of the IIF pattern, titer, and ENA results (Figure 1A). These patients would require monitoring and followup testing to determine if their ENA status changes.

In the second algorithm, anti-DFS70 should be tested on all ANA-positive samples. Then again, if the anti-DFS70 result adds no value to the diagnostic interpretation of ENA-positive samples, then there is no need to test for it in patients with a positive ENA (Figure 1B). Further studies

Table 1. Test results and clinical details of anti-DFS70-positive patients.

\begin{tabular}{|c|c|c|c|c|}
\hline Sample & $\begin{array}{l}\text { ANA-2 IIF Pattern Operator } \\
\text { Interpretation LIU } \\
\quad(\text { Cutoff }=48)\end{array}$ & $\begin{array}{l}\text { ANA ELISA/ } \\
\text { ANA } \\
(\text { Cutoff }=1.0)\end{array}$ & $\begin{array}{l}\text { Anti-DFS70 } \\
\text { CIA/CU } \\
\text { (Cutoff }=20)\end{array}$ & Clinical Details \\
\hline 2 & Speckled (438) & Positive (2.75) & $>450$ & $\begin{array}{l}\text { Non-SARD. Recurrent blistering. Previous } \\
\text { ANA-positive (homogenous) and anti-Scl-70. }\end{array}$ \\
\hline 5 & Unrecognised/negative (24) & Positive (1.06) & 119 & $\begin{array}{c}\text { Non-SARD. Primary Raynaud, Previous } \\
\text { ANA-positive (homogenous). }\end{array}$ \\
\hline 6 & Homogenous (215) & Positive (3.5) & 52 & $\begin{array}{l}\text { Non-SARD. Epilepsy, } 2 \times \text { miscarriages. Positive } \\
\text { anticardiolipin, positive lupus anticoagulant. Previous } \\
\text { ANA-positive (speckled), ENA-negative. }\end{array}$ \\
\hline
\end{tabular}

DFS: dense fine speckled; ANA: antinuclear antibodies; LIU: light intensity units; CIA: chemiluminescence immunoassay; CU: chemiluminescent units; SARD: systemic autoimmune rheumatic diseases; ENA: extractable nuclear antigen; SLE: systemic lupus erythematosus.

Personal non-commercial use only. The Journal of Rheumatology Copyright $\odot$ 2018. All rights reserved. 


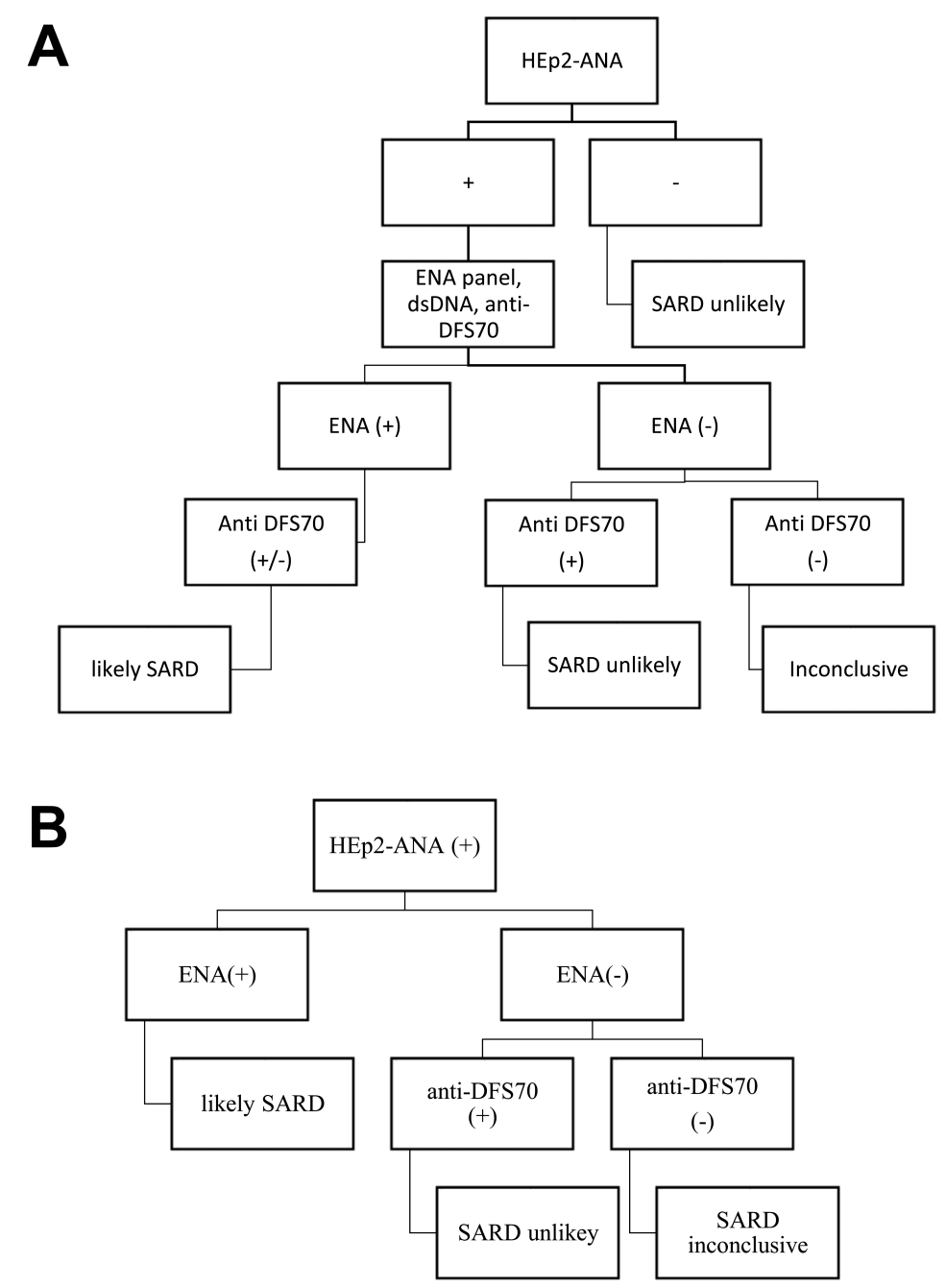

Figure 1. (A) Proposed new ANA result interpretation algorithm that includes the anti-DFS70 results. (B) Proposed new ANA test algorithm considering anti-DFS70 antibodies on all ANA-positive samples. ANA: antinuclear antibodies; DFS: dense fine speckled; ENA: extractable nuclear antigen; SARD: systemic autoimmune rheumatic diseases.

with a larger sample size and inclusive of other laboratories should be done to validate and confirm our findings, and to standardize interlaboratory protocols.

STACEY LUCAS, MMLS, North Shore Hospital Laboratory, Waitemata District Health Board (WDHB); WEE LEONG CHANG, MMLS,

AUT-Roche Diagnostics Laboratory, Auckland University of Technology, Private Bag 92006; FABRICE MERIEN, DVM, PhD, AUT-Roche Diagnostics Laboratory, Auckland University of Technology, Private Bag 92006, Auckland, New Zealand. This research project was funded by the AUT-Roche Diagnostics Laboratory and supported by WDHB, Auckland, New Zealand. Address correspondence to Dr. F. Merien, Auckland University of Technology, School of Science, 34 St Paul St., Auckland 1142, New Zealand.

E-mail: fmerien@aut.ac.nz

\section{REFERENCES}

1. Smeenk RJ. Antinuclear antibodies: cause of disease or caused by disease? Rheumatology 2000;39:581-4.

2. Copple SS, Jaskowski TD, Giles R, Hill HR. Interpretation of ANA indirect immunofluorescence test outside the darkroom using NOVA view compared to manual microscopy. J Immunol Res 2014;2014:149316.

3. Swaak AJ. [Diagnostic significance of antinuclear antibodies in clinical practice]. [Article in Dutch] Ned Tijdschr Geneeskd 2000;144:585-9.

4. Copple SS, Giles SR, Jaskowski TD, Gardiner AE, Wilson AM, Hill HR. Screening for IgG antinuclear autoantibodies by HEp-2 indirect fluorescent antibody assays and the need for standardization. Am J Clin Pathol 2012;137:825-30.

5. Kumar Y, Bhatia A, Minz RW. Antinuclear antibodies and their 
detection methods in diagnosis of connective tissue diseases: a journey revisited. Diagn Pathol 2009;4:1.

6. Phan TG, Wong RC, Adelstein S. Autoantibodies to extractable nuclear antigens: making detection and interpretation more meaningful. Clin Diagn Lab Immunol 2002;9:1-7.

7. Miyara M, Albesa R, Charuel JL, El Amri M, Fritzler MJ, Ghillani-Dalbin P, et al. Clinical phenotypes of patients with anti-DFS70/LEDGF antibodies in a routine ANA referral cohort. Clin Dev Immunol 2013:703759.
8. Gundín S, Irure-Ventura J, Asensio E, Ramos D, Mahler M, Martínez-Taboada V, et al. Measurement of anti-DFS70 antibodies in patients with ANA-associated autoimmune rheumatic diseases suspicion is cost-effective. Auto Immun Highlights 2016;7:10.

J Rheumatol 2018;45:2; doi:10.3899/jrheum.170849 